\title{
UNE "POLÉMIQUE DÉFENSIVE" : LE MONDE FACE AUX ACCUSATIONS DE PIERRE PÉAN ET PHILIPPE COHEN
}

\author{
Raphaël Micheli ${ }^{1}$
}

Dans un article paru en 1985, Artur Greive suggère que les travaux sur la polémique privilégient trop souvent ce qu'il appelle 1'"explication (...) à sens unique". Une telle explication tend, selon lui, à présenter la polémique uniquement comme "une arme d'attaque". Or, affirme-t-il,

Pour qu'il y ait polémique, il faut au moins deux personnes, et nous savons que quiconque est attaqué, ou se croit attaqué, a pour habitude de se défendre. Il est donc grand temps de nous demander si, à côté des signes caractéristiques de la polémique offensive, il n'en existe pas aussi pour la polémique défensive ${ }^{2}$.

Je me propose d'examiner ici un exemple particulièrement frappant de "polémique défensive". Il s'agit de la réaction du journal Le Monde, dans son édition du 7 mars 2003, suite à la parution en

1 Université de Lausanne, Centre de Recherche en Linguistique Textuelle et Analyse des Discours.

2 A. Greive, "Comment fonctionne la polémique ?", in G. Roellenbleck (dir.), Le discours polémique, Paris/Tübingen, Jean-Michel Place/Gunter Narr Verlag, 1985.

Recherches en communication, $\mathrm{n}^{\circ} 20(2003)$. 
librairie de l'ouvrage de Pierre Péan et Philippe Cohen : La face cachée du Monde. Du contre-pouvoir à l'abus de pouvoir ${ }^{1}$. Dans le cadre d'une réflexion sur la polémique journalistique, le choix d'un tel sujet présente l'intérêt de varier quelque peu la perspective : il s'agit, en effet, de voir ce qui se passe lorsqu'un organe de presse devient la cible même d'une charge polémique, et non plus son simple lieu d'expression ou relais, comme c'est habituellement le cas.

La notion de "polémique défensive" est d'apparence quelque peu oxymorique. Je m'en servirai surtout afin de mettre en évidence la profonde ambiguiité de la réaction du grand quotidien face à l'attaque qu'il subit. On peut, suivant Artur Greive, admettre en première approximation que "l'arsenal des formes linguistiques de la polémique défensive coïncide en grande partie avec celui de la polémique offensive"2. Je défendrai pour ma part l'hypothèse que la "polémique défensive" ne va pas sans certains paradoxes spécifiques d'ordre interactionnel et rhétorico-argumentatif. S'agit-il pour les responsables du Monde de "polémiquer" ? Au contraire : l'important travail de recadrage de l'interaction en cours suggère une volonté très nette de se placer hors de l'échange polémique proprement dit -que ce soit, on le verra, par la sélection drastique des allocutaires légitimes ou par l'explicitation du macro-acte de discours censément accompli ( $\$ 1$ et 2). Toutefois, les responsables du Monde doivent bon gré mal gré se "défendre" : il importe, dans une telle situation de crise, de mettre rapidement fin aux rumeurs et de rétablir l'image de marque du quotidien. Cette "défense" est, on le constatera, éminemment paradoxale. Elle juxtapose en effet deux stratégies discursives distinctes et, au premier abord, mutuellement exclusives : la disqualification a priori du discours adverse et sa réfutation argumentée. Je décrirai cette "défense" dans sa dualité. J'en esquisserai la topographie -les lieux stratégiques du journal où elle se déploie préférentiellement- et la très large palette rhétorique -de l'usage d'un lexique infamant à la contre-argumentation méthodique (§3). Je reviendrai, en fin de parcours, sur les ambiguïtés, voire sur les apories de la "polémique défensive" (\$4).

1 Paris, Mille et Une Nuits, 2003.

2 A. GREIVE, op. cit. 


\section{Une polémique qui ne se dit pas}

Il est rarement avantageux, pour un locuteur, de désigner ou de qualifier explicitement sa propre prise de parole par le substantif ou l'adjectif "polémique". Cela tient sans doute à la charge évaluative dont ce terme est porteur. Christian Plantin opère une distinction nécessaire entre un usage "au premier degré" du lexème "polémique" et un usage "méta". Dans le premier cas, il s'agit d'une "utilisation directe" qui, de nos jours, est ressentie comme disqualifiante pour le(s) discours concerné(s): Plantin affirme que "désigner un débat comme polémique, c'est s'en distancier"1. Dans le second cas, en revanche, le terme "polémique" désigne non plus une ou plusieurs interaction(s) verbale(s) particulière(s), mais bien le fait même de polémiquer, c'est-à-dire, suivant l'usage contemporain passablement affadi du terme, le fait de débattre ou de confronter des idées avec une certaine vigueur. Des syntagmes figés comme "les vertus de la polémique" ou encore "la salubrité de la polémique" véhiculent, comme le souligne Plantin, un "point de vue positif"2.

Il est remarquable que, dans notre corpus, les parties en présence évitent toutes deux l'usage "au premier degré" du terme "polémique". Observons ce passage tiré de l'introduction de La face cachée du Monde :

Parler de scandale à propos du nouveau Monde ne relève pas d'une simple polémique de journalistes. La place prise par le quotidien dans la vie et le fonctionnement de la République est désormais décisive, car il terrorise les hommes politiques, inquiète les responsables économiques, intimide les éditeurs, les intellectuels et les syndicalistes. Au cœur du dispositif médiatique français, il influence, neutralise ou tétanise la plupart des autres médias. (...) C'est parce que [l'imposture du Monde] entraîne une véritable dégradation de la vie démocratique de notre pays que nous avons décidé de lever le voile à son sujet. (pp. 19-20).

Il y a ici une volonté très nette de s'affranchir des connotations négatives associées au terme "polémique". Il faut, à ce stade, rappeler que ce dernier subit depuis plusieurs années une extension notable de

1 C. Plantin, "Des polémistes aux polémiqueurs", in G. Declerq, M. Murat et J. DANGEL (dir.), La parole polémique, Paris, Honoré Champion, 2003, pp. 377-409 (p. 401).

2 Ibid. 
sa portée référentielle. Comme le fait remarquer Plantin sur la base d'un corpus journalistique, la "condition intuitive" selon laquelle la désignation "polémique" serait exclusivement réservée à "des questions [à] enjeux" tend, de nos jours, à ne plus être remplie. En ce sens, il n'est plus certain que le terme "polémique" implique, de par son signifié même, un référent à caractère "non-prosaïque"'. On comprend mieux, dès lors, l'usage partiellement dénégatoire du terme qui est celui de Cohen et de Péan. En affirmant que leur entreprise "ne relève pas d'une simple polémique de journalistes", les auteurs tentent de libérer leur prise de parole du caractère local, circonscrit, voire personnel que peuvent revêtir les règlement de comptes entre acteurs d'un même champ d'activité sociale. L'énumération des diverses catégories professionnelles et socio-culturelles placées sous l'influence néfaste du Monde a précisément pour but d'investir le propos d'une dimension généralisante -le livre ne concerne pas uniquement un quotidien, mais bien l'ensemble de la vie médiatique, politique, économique et intellectuelle française- et, pourrait-on ajouter, d'une dimension désintéressée -les auteurs ne sont pas mûs par le ressentiment ou l'animosité envers des confrères, mais bien par le souci de la "vie démocratique". La désignation de la prise de parole par le terme "polémique" laisse ainsi logiquement sa place à une désignation par le terme plus noble d'"enquête".

La thématisation directe de la polémique s'apparente ainsi, chez Cohen et Péan, à une forme de dénégation. Cependant, leurs propos introductifs présentent l'une des caractéristiques les plus frappantes des messages polémiques que Catherine Kerbrat-Orecchioni décrit ainsi : "Le discours polémique est un discours disqualifiant, c'est-àdire qu'il attaque une cible[.] [L'] identification de la cible, et du discours comme polémique, vont absolument de pair"2. De manière significative, le livre s'ouvre sur le constat que Jean-Marie Colombani, Alain Minc et Edwy Plenel ${ }^{3}$ ont "créé un nouveau Monde" (p. 11), énoncé habilement étayé par les déclarations des principaux intéressés eux-mêmes. Le reste de l'introduction -et du livre-consiste, on s'en doute, à inverser l'orientation argumentative positive que l'on pourrait prêter à cet énoncé. A la fin de l'Avertisse-

Ibid., p. 387.

2 C. Kerbrat-Orecchioni, "La polémique et ses définitions", in Le discours polémique, Lyon, PUL, 1980, pp. 3-40 (pp. 12 et 26).

3 Respectivement directeur de la publication, directeur de la rédaction et président du Conseil de surveillance du Monde. 
ment, Cohen et Péan affirment que leur "choix est fait : pour le Monde, contre ceux qui l'ont emmené là où il en est aujourd'hui" (p. 23).

On peut ainsi, à partir de la lecture de cette introduction, inférer un schéma de communication typiquement polémique où se retrouvent les actants suivants : un énonciateur (le "polémiqueur") qui, par son discours, vise une cible clairement identifiée qu'il s'agit de "disqualifi[er]". Il faut ici souligner que cette relation n'est aucunement binaire : elle s'enrichit de la présence d'un "tiers"' que le polémiqueur inclut plus ou moins explicitement dans l'ensemble de ses allocutaires. Cohen et Péan précisent pour leur part que le livre "s'adresse prioritairement à celles et à ceux qui furent [les] lecteurs fervents et attentifs [du Monde] ou qui en sont restés les acheteurs alternativement résignés et indignés..." (p. 22). On peut, à ce stade, représenter ainsi le dispositif polémique mis en place par Cohen et Péan :

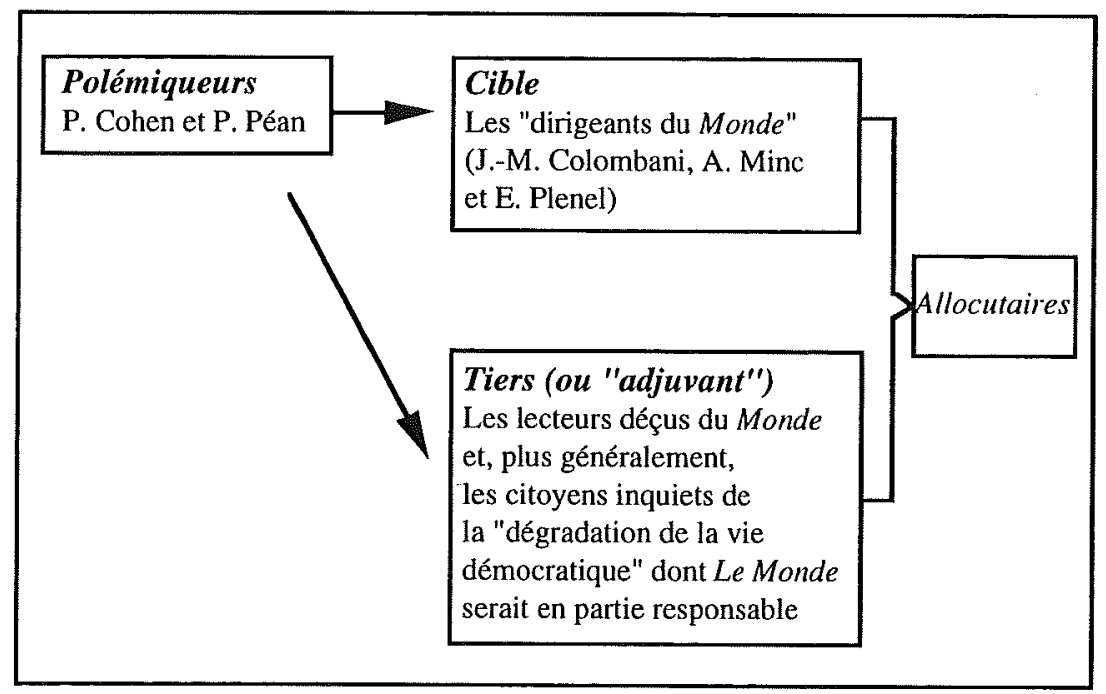

1 Christian Plantin fait usage de cette notion pour définir l'un des "rôles actanciels" de l'interaction argumentative (voir par exemple p. 381). Il me semble que la figure du "tiers" joue à plein dans toute interaction polémique où le polémiqueur attaque sa cible devant des spectateurs réels ou virtuels. Cette dimension triangulaire de l'interaction polémique apparaît également dans le modèle proposé par Catherine Kerbrat-Orecchioni , cette dernière évoquant les "adjuvants" de la polémique qui peuvent être inclus -ou non-dans l'ensemble des allocutaires. 
On remarquera que les auteurs ne visent pas à l'exclusion pure et simple des "dirigeants actuels du Monde" hors du cercle de leurs allocutaires. Ils soulignent qu'ils ont pris contact avec eux lors de la rédaction de leur livre -sans succès. Ils affirment en outre être conscients que ledit livre "ne manquer[a] pas de [leur] déplaire" (p. 21). Il n'y a ainsi pas, on le voit, d'incompatibilité a priori entre le rôle actanciel de cible et le statut d'allocutaire du message polémique. En ce sens, on peut dire, suivant Catherine Kerbrat-Orecchioni, que dans le cas qui nous intéresse, "la cible est admise comme l'un, au même titre que les autres, des destinataires"'.

Tournons-nous à présent vers la réaction officielle du Monde. Peut-on, au sein de ce contre-discours, postuler une simple réversibilité des rôles? Il paraît prévisible que le "polémiqueur" initial va devenir à son tour la "cible" du contre-discours. Cette "cible" nouvellement constituée figure-t-elle toutefois parmi les allocutaires explicitement désignés ? Je défendrai ici l'hypothèse suivante : la réaction du Monde procède moins d'une simple inversion des rôles propres au dispositif polémique que d'un réagencement en profondeur de ce dispositif, notamment au niveau de la sélection des allocutaires légitimes et du macro-acte de discours censément accompli.

\section{Un recadrage de l'interaction en cours}

L'éditorial du 7 mars 2003 est amorcé en Une et porte la signature de Jean-Marie Colombani, directeur de la publication. On reconnaît là les signes d'une situation exceptionnelle, les éditoriaux du Monde étant d'ordinaire dépourvus de signature et situés en milieu de cahier dans la rubrique "Horizons". Par ailleurs, cet éditorial est coiffé d'un titre aux allures de forme d'adresse : "A nos lecteurs". On peut, au premier abord, être surpris par ce choix. La face cachée du Monde est déjà en librairie depuis le 26 février : ne serait-il pas dans ces conditons plus logique que l'énoncé-titre prenne comme référent le livre de Cohen et Péan, ce afin de situer l'objet du discours de manière immédiate et parfaitement explicite ? En réalité, ce choix de titre participe d'un recadrage de la situation d'interaction dont il importe de saisir les enjeux. En intitulant l'éditorial "A nos lecteurs", le directeur de la publication désigne d'une part explicitement celles et ceux qu'il considère comme ses allocutaires légitimes et, d'autre

1 C. Kerbrat-Orecchion, "La polémique et ses définitions", op. cit., p. 28. 
part, plus implicitement, ceux qu'il juge indignes de ce statut. On peut suivre ce processus de hiérarchisation des allocutaires, voire d'exclusion de certains d'entre eux.

Comme le suggère habilement J.-M. Colombani, il ne s'agirait pas tant d'une exclusion opérée par la rédaction du Monde que d'une auto-exclusion imputable à Cohen et Péan eux-mêmes. La nature de leur discours, qu'il s'agisse de son genre (la "calomnie") ou de sa visée pragmatique dominante ("salir, détruire"), interdit de facto à ses auteurs de recevoir le statut d'allocutaires. Il y a, de la part de la rédaction du Monde, un refus de se placer dans le cadre commun d'une interaction verbale, mieux, d'un échange avec Cohen et Péan. Ce refus transparaît nettement lorsque les responsables du Monde explicitent le macro-acte de discours ${ }^{1}$ qui, selon eux, confère son unité aux différents articles de l'édition du 7 mars. Considérons les exemples suivants :

Tandis que ses avocats s'apprêtent à déposer des plaintes en diffamation publique, notre journal a décidé d'informer ses lecteurs sur sa stratégie économique, ses relations avec ses partenaires professionnels et le strict contrôle de ses comptes. (Chapeau de l'article "Le Monde oppose les faits aux accusations calomnieuses", 7 mars 2003, p. 18, je souligne).

Le Monde aurait donc fait l'objet d'une enquête et devrait répondre au plus vite sur les faits qu[e cette enquête] aurait révélés. De notre point de vue, [une telle affirmation] n'a pourtant pas la force d'une évidence. (Attaque de l'article " $L e$ Monde oppose les faits aux accusations calomnieuses", 7 mars 2003, p. 18, je souligne).

Notre devoir cependant est de revenir vers notre seul juge, celui pour lequel une collectivité se dévoue sans compter, à savoir nos lectrices et nos lecteurs, qui nous ont tant de fois manifesté leur confiance et leur solidarité. (...) En leur donnant non pas une réponse, mais les éléments d'information indispensables tant sur la marche réelle de l'entreprise Monde que sur la couverture de l'actualité qu'il a assurée au fil des

1 Dans le cadre de sa pragmatique textuelle, Jean-Michel Adam rappelle que "[c]omprendre un texte, c'est [en partie] pouvoir répondre à une question pragmatique : pourquoi, pour accomplir quel but $(. .$.$) ce texte a-t-il été produit ?$ Comprendre l'action langagière engagée (macro-acte de discours implicite ou explicite), c'est une autre façon de résumer un texte et donc de l'interpréter dans sa globalité." (J.-M. ADAM, Linguistique textuelle. Des genres de discours aux textes, Paris, Nathan, 1999, p. 79). 
dernières années, pour répondre à celles et à ceux qui ont pu être troublés par tant de boue déversée sur notre journal. (Le Monde, éditorial, 7 mars 2003, p. 18, je souligne).

Il semble ici y avoir une dichotomie [répondre, réponse / informer, éléments d'information] dont le premier terme est presque systématiquement rejeté par les responsables du Monde. On perçoit chez eux une répugnance certaine à catégoriser le macro-acte de discours comme une "réponse", qui plus est comme une "réponse" $a$ Cohen et Péan. La théorie des actes de discours développée par Daniel Vanderveken ${ }^{1}$ permet de mieux comprendre en quoi "répondre" est, dans le cas qui nous intéresse, jugé inacceptable. Selon D. Vanderveken, le verbe "demander" est, dans ses deux sens ("faire une requête" et "poser une question"), un verbe directif : il a en effet pour but, selon l'expression de J. R. Searle, de "faire faire des choses à autrui"2, c'est-à-dire d'amener l'allocutaire à adopter un certain comportement. Quelle est la nature exacte de ce comportement ? "L'action demandée", poursuit Vanderveken, est "un acte de discours futur adressé au locuteur initial qui soit une réponse à sa question"3. Il est crucial, pour notre propos, de replacer le couple question / réponse dans le cadre des actes de discours directifs. Caractériser la réponse comme une "action" que le questionné accomplit à la demande expresse du questionneur, c'est du même coup y déceler une forme de soumission à autrui. Le fait que cette "action" doive nécessairement prendre la forme d'un discours "adressé au locuteur initial qui soit une réponse à sa question" implique en outre deux conditions : (1) Le "locuteur initial" doit être jugé digne d'être constitué en allocutaire ; (2) Celui qui répond doit accepter que la question "détermine" (ibid.), en partie au moins, la forme et le contenu de sa réponse. Ce sont précisément ces deux conditions qui, aux yeux des responsables du Monde, ne sont pas remplies : (1) Si "réponse" il y a, elle s'adresse exclusivement au "tiers" ("celles et (...) ceux qui ont pu être troublés par tant de boue déversée sur notre journal"), mais en aucun cas aux "polémiqueurs" eux-mêmes ; (2) Les "accusations calomnieuses", pire, la "boue" ne sauraient déterminer le contenu propositionnel d'une réponse docile.

1 D. VANDERVEKEN, Les actes de discours, Liège, Mardaga, 1988.

2 J.R. SEARLe, L'intentionnalité. Essai de philosophie des états mentaux, Paris, Éd. de Minuit, 1985, p. 201.

3 D. VANDERVEKEN, op. cit., p. 183. 
On assiste ici à un spectaculaire recadrage de la situation d'interaction qui implique à la fois une recatégorisation du macro-acte de discours en passe d'être accompli et une discrimination des allocutaires. Il ne s'agit pas de "répondre" aux auteurs de La face cachée du Monde, mais bien, pour reprendre l'un de nos extraits, $d$ " "informer les lecteurs". Le choix du verbe "informer" et, ailleurs, du substantif "information" s'explique probablement par le vernis d'objectivité qui recouvre cette famille de lexèmes. Les responsables s'appuient ici en creux sur une dichotomie encore prégnante dans le monde médiatique actuel : celle qui oppose 1" "opinion" à l' "information". Philippe Breton affirme qu'une "opinion" est "un point de vue qui en suppose toujours un autre possible (...), ou qui, dans un débat, s'oppose à d'autres". La direction du Monde refuse à la fois de présenter son discours comme l'expression subjective d'un "point de vue"-il s'agit, comme l'indique un titre précédemment cité, de livrer des "faits"- et de l'inscrire dans une interaction de type "débat" où elle affronterait des allocutaires jugés légitimes. En ce sens, parler d' "information", et non d'"opinion" ou de "point de vue", participe d'une tentative de désubjectivation de la parole et de clôture de la polémique. L' "information", si elle est assez exhaustive, n'appelle idéalement pas de nouvelles questions. La sélection des "lecteurs" comme unique instance allocutrice légitime ne fait, quant à elle, que s'accentuer au fil de l'éditorial de J.-M. Colombani précédemment cité. L'auteur érige "[les] lecteurs et [les] lectrices [du Monde]" en "seul juge, celui pour lequel une collectivité se dévoue sans compter" (Le Monde, 7 mars 2003, p. 18). Le marquage énonciatif se fait ensuite plus direct : le pronom personnel "vous" vient en effet renforcer l'écho du titre que l'on trouve à l'entame du dernier paragraphe et qui encadre l'éditorial: "A nos lectrices et à nos lecteurs, (...) je peux vous assurer que «nous maintiendrons»" (ibid., je souligne).

On peut schématiser comme suit le réagencement en profondeur du dispositif de communication :

1 P. Breton, L'argumentation dans la communication, Paris, La Découverte, 2001, p. 28. 


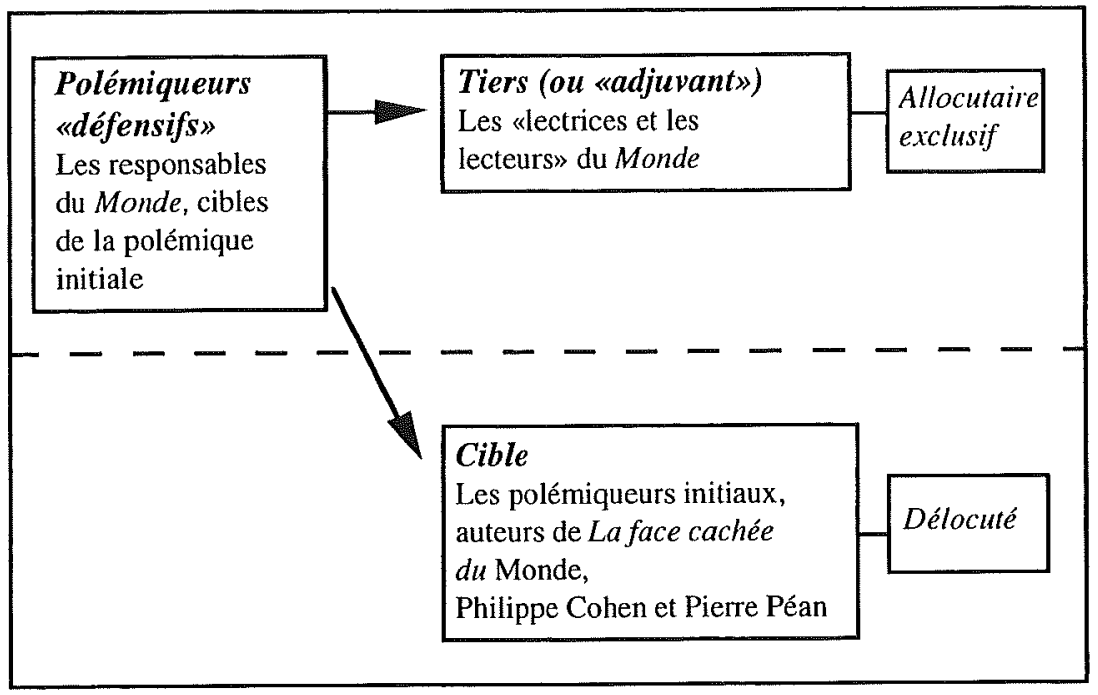

On voit bien, à présent, qu'on ne saurait parler d'une simple réversibilité des places. Le "tiers" se voit ici constitué en instance allocutrice unique qu'il s'agit d' "informer". La cible implicite du contre-discours est, quant à elle, exclue de la sphère d'interlocution ${ }^{1}$ : il ne s'aurait s'agir, on s'en souvient, de lui accorder la faveur d'une "réponse". Cependant, et c'est là toute l'ambiguïté de la réaction du Monde, le discours adverse se voit soumis à un double travail de disqualification et de réfutation. Voyons donc comment se formule cette "réponse" qui ne se dit pas.

\section{Entre disqualification a priori et réfutation argumentée : les paradoxes d'une "réponse"}

"Polémiquer", affirme C. Kerbrat-Orecchioni, "c'est tenter de falsifier (...) la parole de l'autre. Ce qui appelle [une question] : quel est le statut de cet intertexte (...) ?"2. Dans le cas qui nous intéresse, l' "intertexte" reçoit un statut équivoque : l'ouvrage de Cohen et Péan est en effet tout à la fois l'objet d'une disqualification a priori et celui d'une minutieuse réfutation.

1 C'est d'ailleurs l'un des trois cas de figure envisagés par C. Kerbrat-Orecchioni lorsqu'elle s'interroge sur les rapports entre les rôles actanciels et les coénonciateurs des messages polémiques: " La cible [peut être] ouvertement exclue de l'ensemble des destinataires" (C. Kerbrat-OreCCHIONI, op. cit., p. 28).

2 Ibid., p. 10. 
Si l'on adopte une vue d'ensemble du journal, on constate que ces deux procédures se répartissent en zones distinctes. L'éditorial de Jean-Marie Colombani est le lieu par excellence de la disqualification du discours adverse. On peut, dans la même veine, lui ajouter l'article non signé "Le Monde oppose les faits aux accusations calomnieuses". Remarquons l'emplacement liminaire et éminemment stratégique de ces deux textes dans l'espace du quotidien. L'éditorial, amorcé en Une, se termine à la première page de la rubrique "Communication" (p. 18) où il voisine d'ailleurs avec l'article "Le Monde oppose les faits aux accusations calomnieuses". Le reste de cette rubrique (pp. 19-20) est consacrée à un examen critique, puis à une contreargumentation systématique des thèses avancées par Cohen et Péan. Ainsi, pour autant que l'on adopte une lecture linéaire du journal, le travail de disqualification précède celui de réfutation. Là réside sans doute l'un des paradoxes de la réaction du Monde : tout se passe d'abord comme si le discours adverse n'était pas digne que l'on entre en matière, alors qu'il se voit réfuté presque point par point dans les pages qui suivent!

\subsection{Rejet a priori d'un discours odieux et "mal formé"}

Concentrons-nous, pour commencer, sur ce premier moment de disqualification en observant les chaînes désignatives qui prennent pour référents Philippe Cohen et Pierre Péan, leur ouvrage La face cachée du Monde ou encore leurs motifs et leur buts implicites' ${ }^{1}$. La pléthore de ces désignations peut se répartir en quelques grands espaces sémantiques distincts. Celui, tout d'abord, de la violence physique et de la guerre (polemos): on a "fait feu" sur Le Monde avec "une arme : la calomnie" (1); L'ouvrage en cause constitue une "agression"; Ses auteurs sont des "assaillants" qui "veulent" ou "tentent d'abattre" Le Monde et poursuivent, ce faisant, des "buts de guerre" (1). Celui, ensuite, de la folie ou, à tout le moins, d'un grave déficit de rationalité : le "déferlement de haine" est qualifié d' "invraisemblable" (1), les accusations de "folles" (1), sans parler du "délire d'interprétation" (2) qui saisit les auteurs, "inspir[és]" par une étrange "fièvre" (1) dont on peine à cerner l'origine. Ce lexique de 1a

1 Les citations qui suivent sont tirées de l'éditorial "A nos lecteurs" signé par JeanMarie Colombani (abrégé (1) dans le texte) et de l'article "Le Monde oppose les faits aux accusations calomnieuses" (abrégé (2) dans le texte). 
déficience mentale voisine avec celui d'une malhonnêteté intellectuelle qui, cette fois, semble se placer sous le contrôle intentionnel des agents incriminés : il est alors question de "mauvaise foi" (1), d'“arguments biaisés" (1), d'“anecdotes (...) déformées, (...) voire inventées" (2) ; L'intervention de facteurs émotionnels -le "ressentiment" et la "dévotion déçue" (1)- décrédibilisent 1'"enquête" que les deux journalistes prétendent mener. L'absence de tout souci déontologique comme de toute rigueur méthodologique fait du discours adverse un "jeu (...) pervers", un "livre venimeux" où le "poison [est] distillé à chaque page" (1).

Ce très rapide relevé de quelques items lexicaux dévalorisants suggère que l'on a ici affaire à une mise hors-jeu du discours adverse. Cela se voit confirmé par la substance de l'argumentation déployée dans le long article "Le Monde oppose les faits aux accusations calomnieuses". On peut aisément en dégager la trame. Cohen et Péan affirment avoir "utilis[é] les méthodes classiques du "métier"" (p. 22) et désignent explicitement leur propos comme une "enquête". Or, selon les responsables du Monde, leur propos ne répond justement à aucun des critères propres à ce genre journalistique et, de manière plus large, à aucune des "règles professionnelles (...) censément communes [à l'ensemble du monde de la presse écrite]". Pour ne pas rester lettre morte, un tel contre-argument doit se voir étayé par des données factuelles ou, pour reprendre un terme utilisé, par des "preuve[s]". L'administration de la preuve prend, dans le reste de l'article, la forme d'une vertigineuse plongée dans le détail. Les auteurs égrènent un par un les noms propres incorrectement épelés, les erreurs dans les dates, les concepts juridiques obsolètes, voire fictifs...La charge écrasante de la preuve tient ici à son caractère massivement quantitatif, les auteurs de l'article suggérant, de surcroît, qu'il s'agit là d'une "collecte infinie" et d'un "inventaire non exhaustif". Cette plongée dans le détail acquiert sa pleine portée disqualifiante grâce à ce que l'on pourrait appeler un mécanisme de transfert. Qu'est-ce à dire ? L'“inexactitude" décelée dans les parties se voit reportée sur le tout : elle contamine, depuis les fondations, l'ensemble de l'édifice rhétorique. Le topos à l'origine de ce transfert se lit d'ailleurs en toutes lettres dans l'article: "Si [ l'enquête de Philippe Cohen et Pierre Péan] se trompe en détail, comment pourraitelle prétendre avoir raison en général ?".

On comprend, par ce topos, que la plongée dans le détail sert une visée pragmatique radicale: celle d'une disqualification a priori du 
discours adverse. Il faut souligner, à ce stade, qu'on ne saurait identifier cette dernière avec une réfutation du même discours. La réfutation implique à l'évidence que l'Opposant considère l'argument du Proposant ${ }^{1}$ comme digne d'être réfuté. La disqualification, en revanche, aboutit au "reje[t] [du discours adverse]" parce qu'il est "mal formé, quelle que soit la nature de cette malformation (...), ce qui permet de faire l'économie de l'examen de la proposition"2. Dans le cas qui nous intéresse, les responsables du Monde présentent bien le discours de Cohen et Péan comme étant "mal formé" : sa nonconformité aux standards de l' "enquête" journalistique est l'une des causes majeures de son rejet. On pourrait, dès lors, s'attendre à ce que la disqualification aboutisse à un refus d'entrer en matière sinon sur le "détail", du moins sur la substance des "propositions" de l'argumentation adverse (Le Monde aurait été "balladuriste" en 1995, Le Monde offrirait une couverture outrageusement partiale du problème corse, etc.).

\subsection{La contre-argumentation malgré tout}

Le Monde, on l'a déjà compris, ne va pas au bout d'une telle logique disqualifiante. Dans l'édition du 7 mars 2003, la disqualification précède la réfutation proprement dite et lui prépare en quelque sorte le terrain, mais elle ne saurait la remplacer purement et simplement. Esquissons à présent la topographie de cette réfutation.

- La "réponse" du journal, amorcée en Une avec l'éditorial, trouve sa place, on l'a déjà dit, dans la rubrique "Communication". Cette rubrique est d'ordinaire consacrée à la vie des médias -qu'il s'agisse de la presse écrite, de la télévision, de la radio ou encore des nouvelles technologies de l'information online. On ne saurait cependant se contenter de dire qu'il y a simple utilisation d'une rubrique existante : il y a également réappropriation forte de celle-ci par le quotidien. Cela se marque particulièrement au niveau du péritexte. Aux pages 19 et 20, le rubriquage usuel "Communication" devient, à la faveur d'un ajout significatif, "Communication après l'attaque contre Le Monde". La modification du rubriquage est le signe d'une

\footnotetext{
1 Je reprends ici à nouveau les actants de l'interaction argumentative tels que désignés par C. Plantin (op. cit.).

2 Ibid., p. 493.
} 
situation exceptionnelle. De plus, l'ajout vient infléchir la portée référentielle du lexème "Communication". Ce dernier désigne d'habitude le champ d'activités -les médias- que la rubrique prend pour thème. Dans le cas présent, il désigne plutôt, de manière réflexive, l'activité discursive en passe d'être accomplie. En réintitulant la rubrique "Communication après l'attaque contre Le Monde"; les rédacteurs explicitent ce qu'ils font, mieux, ce qu'ils sont en train de faire : communiquer avec leurs lecteurs. Il ne s'agit donc plus de la communication en général -le fait même de communiquer et les moyens techniques que les personnes utilisent pour communiquer-, mais bien d'une communication particulière -à la fois l'action de communiquer quelque chose à quelqu'un et le résultat de cette action. On peut parler ici d'une réappropriation de la rubrique par le biais d'une altération péritextuelle signifiante.

Si l'on se penche maintenant sur l'anatomie des articles, on ne peut manquer d'être frappé par l'extrême visibilité de la contre-argumentation. Les différentes étapes du processus réfutatif se marquent en effet dans chacun des éléments constitutifs de l'article.

- Le titre est polyphonique : il porte la trace explicite du discours accusateur de Cohen et Péan. Comme le rappelle H. Nølke, "[Un] locuteur est à même de mettre en scène des énonciateurs qui présentent différents points de vue. Il peut s'associer à certains énonciateurs tout en se dissociant d'autres"l. Ici, le "point de vue" des auteurs de La face cachée $d u$. Monde est systématiquement invalidé. Le titre "La Corse traitée sans complaisance" (Le Monde, 7 mars 2003, p. 18) met par exemple en scène, pour le nier, le point de vue exprimé dans le chapitre 13 ("Un «Monsieur Corse» nommé Le Monde"); Quant au titre "Le «balladurisme» du Monde en 1995, une mauvaise querelle" (p. 18 également), il évalue négativement le point de vue défendu dans le chapitre 9 ("Journal de campagne"). Le titre n'implique pas forcément de prédication et peut fort bien se constituer de simples syntagmes nominaux : "La hausse des ventes et la baisse de la publicité" (p. 19). Dans ce dernier exemple, la réfutation passe par une permutation des pivots nominaux entre les deux syntagmes. Le point de vue latent soumis à réfutation serait donc quelque chose comme "La baisse des ventes et la hausse de la publicité". On peut, en

1 H. Nølke, "Polyphonie", in D. Maingueneau et P. Charaudeau (dir.), Dictionnaire d' analyse du discours, Paris, Éd. du Seuil, 2002, pp. 444-448 (p. 445). 
s'inspirant de Nølke et en reprenant l'un de nos exemples, représenter ainsi le caractère polyphonique des titres de notre corpus :

\section{"La Corse traitée sans complaisance"}

\section{PdV 1 : "La Corse [a été] traitée [avec] complaisance" $P d V 2$ : "PdV 1 est injustifié"}

Ceci montre bien que "deux points de vue incompatibles cohabitent"l au sein du même énoncé. Ce genre de titre, s'il est polyphonique, est également réfutatif : il encapsule à la fois le point de vue adverse et un point de vue au second degré, qui consiste en une évaluation négative du premier point de vue.

- Situé entre le titre et le corps de l'article, le chapeau revêt d'ordinaire une double fonction, à la fois en amont et en aval : d'une part, il "complète le titre en précisant (...) l'angle qu'a choisi le rédacteur pour traiter [le sujet]" et, d'autre part, il "résume l'information en donnant l'essentiel de ce qu'il faut savoir si l'on doit s'arrêter là" 2 . Ici, le chapeau précise sous quel angle le point de vue adverse, mis en scène polyphoniquement dans le titre, va être déclaré caduc. En ceci, le chapeau "résume" et "donn[e] l'essentiel" de la contre-argumentation qui forme le corps de l'article. Sous le titre "La Corse traitée sans complaisance", on lit par exemple: "Le quotidien a rendu compte régulièrement des exactions nationalistes". L'explicitation du titre s'allie bien ici à une annonce de la contre-argumentation qui va suivre -le corps de l'article fournissant une série d'exemples de prises de positions critiques du Monde envers les attentats perpétrés sur l'île. Ailleurs, sous le titre "Le «balladurisme» du Monde en 1995, une mauvaise querelle", on apprend que "L'ensemble de la couverture de la campagne présidentielle est occulté" dans l'ouvrage de Cohen et Péan.

- L'attaque de 1'article a pour fonction de reprendre et d'expliciter la thèse adverse qui va être soumise à réfutation. Contrairement au titre, elle opère une nette séparation des voix : "Le Monde protégerait les nationalistes corses, au dire de Pierre Péan et Philippe Cohen" et "Selon Pierre Péan et Philippe Cohen, Le Monde

1 Ibid.

2 Y. AGNÈs, Manuel de journalisme. Écrire pour le journal, Paris, La Découverte, coll. "Repères", 2002, p. 149. 
serait devenu le «journal de campagne d'Edouard Balladur»". Le discours adverse peut ici être clairement circonscrit grâce aux procédés de modalisation en discours second ${ }^{1}$ : le conditionnel et les marqueurs d'univers de discours ("Selon..." et "au dire de...") indiquent clairement, en plus des fragments au discours direct, que l'énonciateur n'est pas responsable du contenu propositionnel livré.

- Le corps de l'article est également le corps de la réfutation. Ce qui est en jeu ici, c'est de porter un coup fatal au contenu propositionnel livré lors de l'attaque. Pour cela, les rédacteurs du Monde se placent en amont : ils tentent d'invalider l'enchânement argument(s)-conclusion sur lequel l'adversaire appuie ses thèses accusatrices. La contre-argumentation du Monde prend le plus souvent pour cible le manque de "complétude"2 de l'argumentation adverse : il y aurait, chez Cohen et Péan, un refus tendancieux de considérer l'ensemble des données factuelles à disposition. La contreargumentation stigmatise ici ce que les anglo-saxons appelleraient fallacy of suppressed evidence : "Ignorer délibérément une donnée importante qui, dans un cas particulier, pèse plus lourd que la donnée fournie et entraîne une conclusion différente" 3 . C'est là un leitmotiv de la contre-argumentation. Concernant les relations de connivence qui auraient existé entre le Monde et Jean-Marie Messier, les journalistes visés affirment que Cohen et Péan "occultent (...) de nombreux articles (...) qui sont loin d'être louangeurs" (Article "Les relations avec Vivendi et Jean-Marie Messier", Le Monde, 7 mars 2003 , p. 20). Au sujet de le campagne présidentielle de 1995, ils "occultent l'ensemble de la couverture" en ne retenant qu' "un seul" article sur les 576 que Le Monde dit avoir publiés à ce sujet. On pourrait multiplier les exemples où se lit cette violente dénonciation de la suppressed evidence : les auteurs de La face cachée du Monde "omettent" ou "se gardent de mentionner" des sources et, par

1 Voir à ce sujet les travaux de Jacqueline Authier-Revuz, et notamment "Repères dans le champ du discours rapporté", L'information grammaticale, $\mathrm{n}^{\circ} 55$ et 56 , 1992 et 1993.

2 C'est l'un des quatre "modes" de la contre-argumentation recensés par P.-Y. Brandt et D. Apothéloz (voir C. PLANTIN, "Contre-argumentation", in P. Charaudeau et D. Maingueneau (dir.) Dictionnaire d' analyse du discours, p. 141).

3 "The ignoring of an important piece of evidence, in a particular case, that outweighs the presented evidence, and entails a different conclusion." (D. WALTON, The Place of Emotion in Argument, The Pennsylvania State University Press, 1992, pp. 159-160, ma traduction). 
conséquent, leurs lecteurs ne sont "pas renseignés, contrairement à ceux du Monde".

\section{Les paradoxes de la "polémique défensive"}

L'examen de la réaction du quotidien Le Monde aux attaques du livre de Pierre Péan et de Philippe Cohen a permis de mettre en évidence certains des paradoxes que l'on peut supposer inhérents à la "polémique défensive".

Paradoxe relatif, tout d'abord, au statut du macro-acte de discours accompli et à l'identité des allocutaires légitimes. J'ai tenté de montrer par quelles contorsions rhétoriques les dirigeants du Monde tentent de se dégager d'une "réponse" qu'ils adresseraient à Cohen et à Péan pour privilégier exclusivement, en théorie tout du moins, l'"information" qu'ils entendent donner aux lecteurs. Il y a là un important travail de recadrage de l'interaction en cours qui fait du "tiers" la seule instance allocutrice légitime et, partant, exclut la "cible" de l'ensemble des destinataires. Un tel recadrage participe d'un fantasme de répondre sans répondre ou, plus réalistement, de répondre sans avoir l'air de s'abaisser à répondre. On mesure bien, à présent, l'inconfort ${ }^{1}$ extrême de la posture discursive adoptée par les dirigeants du Monde: il leur faut afficher avec véhémence leur mépris, par définition silencieux, de l'adversaire en ne lui répondant pas -ou, à tout le moins, en disant qu'ils ne lui répondent pas-et, dans le même temps, avancer une réfutation efficace de la charge polémique.

Paradoxe relatif, ensuite, au statut de cette réfutation. Dans un très riche chapitre de La parole pamphlétaire intitulé "Techniques de la réfutation", Marc Angenot affirme d'entrée de jeu qu'"il n'est pas aisé de décider ce qui a une fonction de réfutation dans un écrit polémique". Il ajoute :

Réfuter ne peut se limiter à contre-argumenter une thèse ou à mettre en cause systématiquement l'argumentation adverse. Au sens strict, il est vrai, la réfutation se limite à cela. Cependant, (...), il est difficile de ne pas rapprocher [de la réfutation] toutes sortes de moyens visant [notamment] à

1 Inconfort d'ailleurs stigmatisé par un lecteur du quotidien qui lance l'injonction suivante : "Osez ouvrir un débat sur Le Monde, dans les colonnes du Monde, avec Cohen et Péan" (Courrier des lecteurs, Le Monde, 17 mars 2003, p. 12). 
disqualifier ou invalider l'adversaire, dans sa parole ou sa personnel.

Ce propos paraît d'une grande pertinence pour le cas qui nous a intéressé ici. Dans le cadre de la "polémique défensive" engagée par le Monde dans son édition du 7 mars 2003, réfuter ne peut en effet se "limiter à contre-argumenter". J'ai montré que les zones liminaires -la Une et la première page de la rubrique "Communication" dévolue dans son entier à la "réponse" du quotidien-sont celles d'une disqualification a priori. Cette disqualification passe par l'usage d'un lexique infamant pour référer à l'adversaire, à sa parole, à ses buts et motifs implicites. Elle se traduit aussi, on l'a vu, dans le verdict de non-conformité au genre de l' "enquête" rendu par Le Monde à l'attention du discours adverse. L'aboutissement d'une telle logique disqualifiante serait une fin de non-recevoir ou, en d'autres termes, le refus de se livrer à une réfutation "au sens strict". Les dirigeants du Monde sont probablement conscients que réfuter, c'est encore, selon une belle formule de $\mathrm{M}$. Angenot, "prendre [d'une certaine manière] en considération les thèses qu'[on] attaque"2. Et pourtant... Il y a bien, comme le prouve la structure de base de quelques articles, une réfutation "au sens strict". Le titre, le chapeau, l'attaque et le corps du texte marquent les étapes d'une contre-argumentation méthodique et visible comme telle. On pourrait dire, en fin de compte, que la disqualification prépare et accompagne la réfutation "au sens strict" plutôt qu'elle ne la court-circuite. Elle est, d'une certaine manière, son versant moral : à la discussion critique des arguments, elle ajoute la condamnation éthique d'agents mal intentionnés et prompts à l'imposture. Elle a pour fonction d'incarner la contre-argumentation en remettant en cause l'idée d'un cloisonnement parfaitement étanche entre les niveaux ad rem et ad hominem ${ }^{3}$.

1 M. ANGENOT, La parole pamphlétaire, Paris, Payot, 1982, p. 211.

2 Ibid., p. 216.

3 Je remercie Jean-Michel Adam pour son aide et ses relectures attentives, ainsi que Jérôme David pour ses nombreuses suggestions. 